\title{
Study on fisheye image correction algorithm
}

\author{
Xue Leng \\ School of Photoelectric Engineering, ChangchunUniversity of Science and \\ Technology ,Changchun, 130000,China. \\ lengxue79@126.com
}

Keywords: fisheye image ,distortion, Correction, Matlab

\begin{abstract}
Abstrict. For serious cases fisheye lens distortion, we use fisheye images captured image get a lot of information presented fisheye correction method using Matlab image processing software, the successful implementation of correcting the image distortion. And experiments show the feasibility of the method.
\end{abstract}

\section{Introduction}

Fisheye shot out of the big picture perspective, a large amount of image information, simplifying the panoramic image acquisition process, saving hardware resources. Fisheye lens has a wide application in many ways, but because there is a serious distortion, need to go through before the correction for the human eye.

\section{Correction Method}

\subsection{Fisheye image correction process}

Fisheye image correction process is to capture fisheye images to useful information, image area occupied detected and split it out, and then determine the center and radius of the image, create a conversion equation image coordinate transformation information to determine the relationship, the information of the source image one by one point assigned to the target image, so as to arrive corrected image. Specific correction process shown in Figure 1.

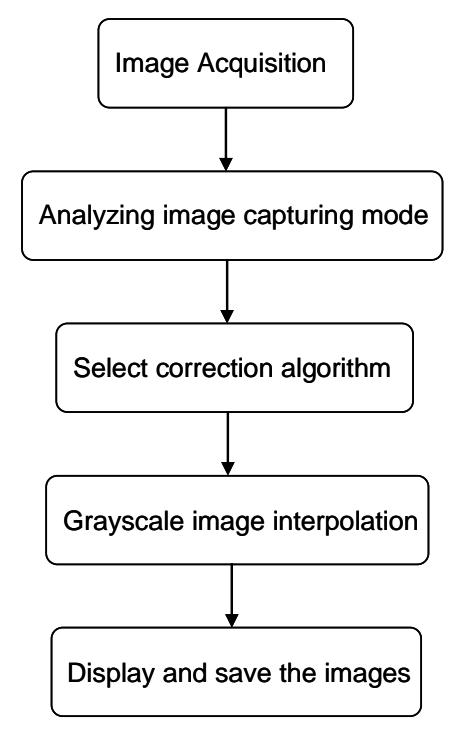

Fig1. Correction flowchart 


\subsection{Correction Algorithm}

In this paper, using concentric segmentation algorithm, the photo information by the fisheye image taken deemed to be the center of a circle of a set of concentric circles, a series of round wire through this correction algorithm converted into a square line image in line with human vision.

Specific correction step:

1 Source image pixel coordinates is $(\mathrm{x}, \mathrm{y})$, the pixel coordinates on the image after the correction is obtained $(\mathrm{u}, \mathrm{v})$, based on characteristics of the image, and can get the coordinate point $(\mathrm{x}, \mathrm{y})$ satisfies the formula (1) and (2) the conditions required.

$$
\begin{aligned}
& \left|x-\frac{w}{2}\right| \leq \frac{\text { width }}{2} \\
& \left|y-\frac{h}{2}\right| \leq \frac{h e i g h t}{2}
\end{aligned}
$$

$w=$ width is a width value of the fisheye image, $h=$ height is a high value of the fisheye image.

The radius of any circle on the image is

$$
R=\sqrt{\left(x-\frac{w}{2}\right)^{2}+\left(y-\frac{h}{2}\right)^{2}}
$$

2 Mapping relationship between the coordinates

$$
\begin{aligned}
& \text { When }\left|\frac{y-\frac{h}{2}}{x-\frac{w}{2}}\right| \leq 1, x \neq \frac{w}{2} \\
& \left\{\begin{array}{l}
u=\sqrt{\left(x-\frac{w}{2}\right)^{2}+\left(y-\frac{h}{2}\right)^{2}} \sin \left(x-\frac{w}{2}\right)+\frac{w}{2} \\
v=\left|\frac{y-\frac{h}{2}}{x-\frac{w}{2}}\right| \sqrt{\left(x-\frac{w}{2}\right)^{2}+\left(y-\frac{h}{2}\right)^{2}} \sin \left(y-\frac{h}{2}\right)+\frac{h}{2}
\end{array}\right.
\end{aligned}
$$

$$
\text { When }\left|\frac{y-\frac{h}{2}}{x-\frac{w}{2}}\right|>1, x \neq \frac{w}{2}
$$

$$
\left\{\begin{array}{l}
u=\left|\frac{x-\frac{w}{2}}{y-\frac{h}{2}}\right| \sqrt{\left(x-\frac{w}{2}\right)^{2}+\left(y-\frac{h}{2}\right)^{2}} \sin \left(x-\frac{w}{2}\right)+\frac{w}{2} \\
v=\sqrt{\left(x-\frac{w}{2}\right)^{2}+\left(y-\frac{h}{2}\right)^{2}} \sin \left(y-\frac{h}{2}\right)+\frac{h}{2}
\end{array}\right.
$$

When $x=\frac{w}{2}$

$$
\left\{\begin{array}{l}
u=\frac{w}{2} \\
v=y
\end{array}\right.
$$

Applying the correction algorithm, the original pixel image by coordinate transformation to transform the pixels on the image after the correction, and the image information assigned to the new image, which can be utilized circle image segmentation algorithm corrected. 

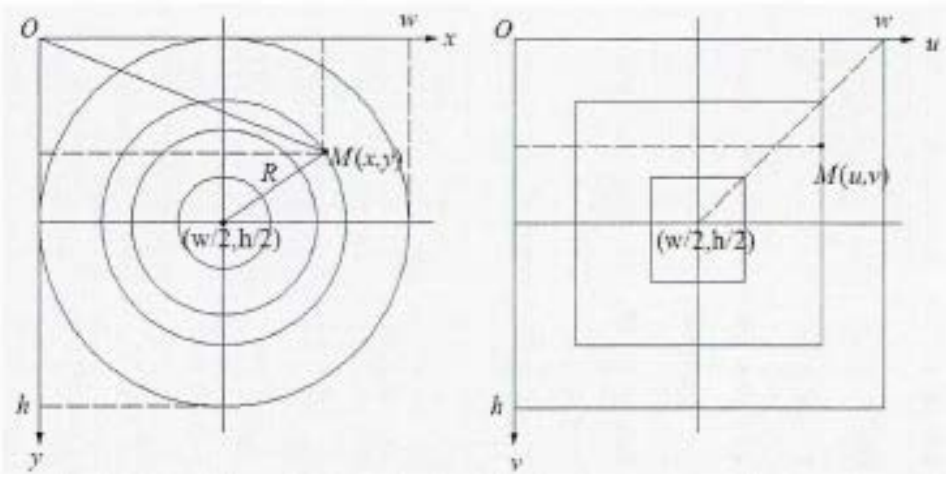

Fig2. The schematic diagram of circle segmentation algorithm

\subsection{Experimental results}

Application round segmentation algorithm, fisheye images for any capturing angle is corrected, the original image is Fig3, Corrected image of is Fig4.

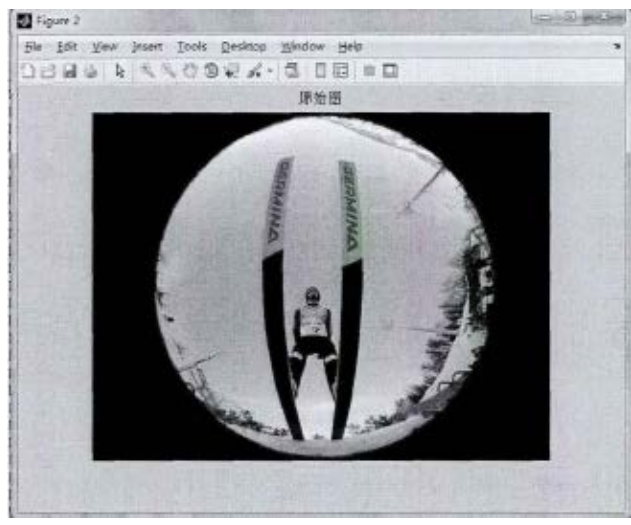

Fig3 Fisheye image

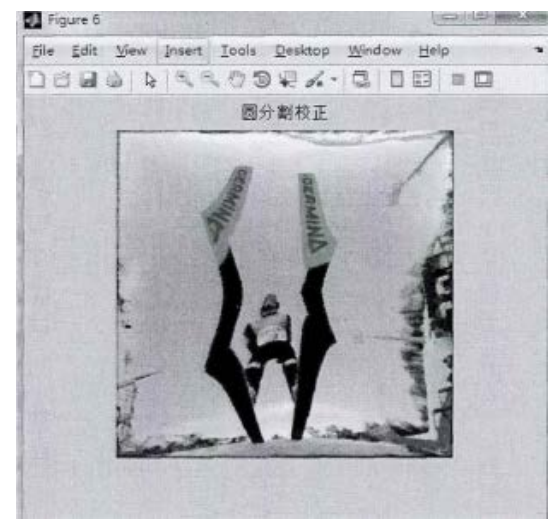

Fig4 Corrected image

Application of circular fisheye corrected image segmentation algorithm, requires that the source image capture image information is a full circle, you can make the correction better. And observed for the four regional eleven upper left, upper right, lower left, lower right four directions of information extracted a region-specific information provided feasible.

\section{Summary}

By Matlab software programming, Achieved the application of the correction algorithm. Confirmed by experiment segmentation for circular fisheye images captured can be applied arbitrarily. Using this method for correcting distortion of the image captured, less demanding on a fisheye lens that can be corrected for any fisheye image.

\section{References}

[1] Jiesong D, Using of fisheye lens to generate a panoramic image method. Journal of Engineering Graphics.Vol15(2010)No.1,P135-138.

[2] Jingjing Y, Based on the geometric properties of the fish-eye image correction algorithm. Computer Engineering.Vol28(2012)No.3,P203-205.

[3] Wei X. Fisheye thematic generalization. Digital Photography.Vol2(2010)No20,P 67-71.

[4] Ling Y. Fisheye image correction Jingwei mapping design method. Journal of Engineering Graphics.Vol20(2010)No6,P19-22. 